\title{
Evaluation of factors leading to anion gap re-opening in adults with diabetic ketoacidosis
}

\begin{abstract}
Purpose: The purpose of this quality improvement project is to identify factors leading to gap re-opening.

Methods: This study was a single-centered, retrospective chart review. The population included all adult patients admitted to a large academic medical center from December 2014 to November 2015 from the emergency department to an adult inpatient floor with a primary diagnosis of diabetic ketoacidosis. The primary efficacy endpoint included gap closure of 12 or less. The primary safety endpoint included gap reopening, defined as an anion gap $>15$.
\end{abstract}

Results: Out of the 30 patients evaluated, 21 patients experienced anion gap reopening while 9 patients did not. The group without anion gap re-opening was younger $(\mathrm{p}=0.276)$, had lower median serum blood glucose on admission $(\mathrm{p}=0.192)$, and lower anion gap on admission $(\mathrm{p}=0.029)$. The length of stay was longer by one day in the anion gap re-opening group versus the group without anion gap re-opening $(\mathrm{p}=0.313)$ and patients without anion gap re-opening had a higher percentage of patients whose anion gap initially closed to $12(\mathrm{p}=0.116)$.

Conclusion: Higher anion gap on admission is a significant predictor of anion gap reopening to greater than 15 . The study may have been underpowered and thus unable to identify other statistically significant factors leading to anion gap re-opening. However, there may be factors such as increased age and clinical targeting of a more restrictive anion gap goal, with clinical significance, in predicting anion gap reopening in diabetic ketoacidosis.

Keywords: diabetes, insulin, diabetic ketoacidosis, anion gap, adult patients, insulin omission, infection, acute cardiovascular event, acute endocrine events, surgical procedures
Volume 5 Issue 3 - 2017

Kathleen K Adams, Gurinderpal Doad, Ginger E Rouse

Department of Pharmacy, Yale New Haven Hospital, USA

Correspondence: Kathleen Adams, Department of Pharmacy, Yale New Haven Hospital, USA, New Haven CT, USA, Tel 7039444400, Email Kathleen.k.adams@gmail.com

Received: March 30, 2017| Published: May 01, 2017
Abbreviations: DKA, diabetic ketoacidosis; AGRO, anion gap re-opening; ADA, american diabetes association; CPOE, computerized physician order entry

\section{Introduction}

\section{Background}

Diabetic ketoacidosis (DKA) is a rare yet serious hyperglycemic complication of diabetes. In 2009, there were 140,000 hospitalizations for DKA with an average hospital stay of 3.4days. Common precipitating factors include insulin omission, infection, acute cardiovascular event, acute endocrine events, and surgical procedures. The majority of patients experiencing DKA are between the ages of 45 and 65 , with two-thirds of patients with a diagnosis of type one diabetes. ${ }^{1,2}$

DKA occurs as a result of insulin deficiency and increased counter-regulatory hormones. These metabolic alterations lead to lipolysis and hepatic fatty acid oxidation to ketone bodies, leading to ketonemia and metabolic acidosis. DKA is diagnosed based on decreased arterial $\mathrm{pH}$, a serum bicarbonate less than $18 \mathrm{mEq} / \mathrm{L}$, presence of urine and serum ketones, and an anion gap $>12$. American Diabetes Association recommended management of DKA includes treatment with intravenous crystalloid fluids and providing regular insulin while managing electrolyte derangements. Recommended insulin dosing regimens include 0.1 unit $/ \mathrm{kg}$ IV bolus with $0.1 \mathrm{unit} / \mathrm{kg} /$ hr IV continuous insulin infusion or $0.14 \mathrm{units} / \mathrm{kg} / \mathrm{hr}$ without a bolus. Unless the episode of DKA is mild, patients should be treated with continuous intravenous insulin until blood glucose $<200 \mathrm{mg} / \mathrm{dl}$ and two of the following criteria are met: serum bicarbonate $\geq 15 \mathrm{mEq} / \mathrm{L}$, venous $\mathrm{pH}>7.3$, or anion gap $\leq 12 \mathrm{mEq} / \mathrm{L}$. At this point, the patient may be transitioned to subcutaneous insulin therapy. ${ }^{3}$

The anion gap is an approximate measurement of ions, and an elevated anion gap will occur when there is an excess of anions to cations in the blood. In DKA, an elevated anion gap indicates that lactate is continuing to be produced and thus, the body has not yet been able to achieve normal insulin-glucose regulation. Closure of the anion gap is a marker utilized by ADA to indicate that DKA has resolved. ${ }^{4}$ Literature evaluating diabetic ketoacidosis is limited and has yet to evaluate factors leading to anion gap re-opening (AGRO).

\section{Objective}

The purpose of this quality improvement project was to identify factors leading to anion gap-reopening as well as areas of improvement in the glycemic management of patients with diabetic ketoacidosis at a large academic medical center. The primary objective was incidence of AGRO, defined as an anion gap $>15$. Anion gap $>15$ was chosen as this was defined as the upper limit of normal within our computerized physician order entry (CPOE) system, despite the American Diabetes Association (ADA) guidelines recommendation to target an anion gap of $10-12$ 


\section{Materials and methods}

This study was a single-centered, retrospective chart review, quality improvement study, and thus did not require review from the Institutional Review Board. The population included all adult patients age 18 years and older that were admitted to our large academic medical center from December 2014 to November 2015. Patients were included if they were admitted from the emergency room to an adult inpatient floor; were 18years of age and older; had a primary diagnosis of diabetic ketoacidosis (DKA); and initiated on a providerdriven insulin infusion. Primary diagnosis was determined by ICD 9 or 10 diagnostic codes. The first thirty patients that met inclusion criteria were evaluated. Patients were excluded if they left against medical advice; had a different primary diagnosis listed in the medical chart besides DKA; were admitted to a pediatric floor; or the treating team utilized a nursing-driven insulin protocol instead of the providerdriven insulin protocol. Provider-driven insulin infusions were defined as insulin infusions managed by providers in accordance with our institution's Adult DKA Management Guideline. This guideline contrasts our institution's nursing driven insulin infusion protocol, which is utilized to manage non-diabetic ketoacidosis hyperglycemia. This nurse-driven protocol is glucose driven as opposed to weightbased, thus there is a possibility of under-dosing. The Adult DKA Management Guideline recommends an initial bolus of $0.1 \mathrm{units} / \mathrm{kg}$ (usually 10units) followed by an insulin infusion of 0.1 units $/ \mathrm{kg}$ (max $15 \mathrm{units} / \mathrm{hr}$ ). The insulin infusion is then adjusted hourly based on rate of change of blood glucose and other clinical findings.

The primary safety endpoint included incidence of AGRO (defined as an anion gap of greater than 15, after having achieved an anion gap of less than or equal to 15), while secondary safety endpoints included median time to anion gap re-opening, highest documented anion gap re-opening, and hypoglycemic events defined as any blood glucose $\leq 70 \mathrm{mg} / \mathrm{dL}$. Efficacy endpoints included gap closure of less than or equal to 12 . Secondary efficacy endpoints included accurate dosing of insulin and accurate transition from parenteral to subcutaneous basal insulin. Accurate dosing and transition were defined by ADA guideline criteria below.

Definitions of various DKA assessments were based on ADA guideline criteria. Accurate insulin infusion was defined as 0.1 units/ $\mathrm{kg}$ IV insulin bolus followed by 0.1 units $/ \mathrm{kg} /$ hour IV continuous infusion or 0.14 units $/ \mathrm{kg} /$ hour IV insulin continuous infusion if no bolus was administered. Appropriate intravenous and subcutaneous insulin overlap was defined as an overlap of 1-2hours. ${ }^{5}$ The Student's T-test was utilized to evaluate continuous data, while Fisher's exact test was utilized to evaluate nominal data.

\section{Results and discussion}

\section{Results}

30 patients met inclusion criteria and were evaluated. 21 patients experienced AGRO while 9 did not. Baseline characteristics that were similar between both groups including percentage of males $(\mathrm{p}=0.443)$, body mass index $(\mathrm{p}=0.715)$, weight $(\mathrm{p}=0.810)$, type 1 diabetes $(p=0.431)$, new onset diabetes $(p=0.640)$, hemoglobin A1C $(p=0.630)$, and use of outpatient oral hypoglycemic agents $(p=1.000)$. The majority of patient received care on general medicine and step down units, with no difference between the two groups $(\mathrm{p}=1.000)$. The group without AGRO was younger (median age 25 vs. 40 years; $p=0.276$ ), with a lower median serum blood glucose on admission $(492 \mathrm{mg} / \mathrm{dL}$ vs. $573 \mathrm{mg} / \mathrm{dL} ; \mathrm{p}=0.192)$, and lower anion gap on admission ( 22 vs. $27 ; \mathrm{p}=0.029)$. The length of stay was greater in the AGRO group versus the group without AGRO (4 vs. 3 days; $\mathrm{p}=0.313$ ). However, only differences in anion gap on admission were statistically significant (Table 1).

Patients were evaluated based on accurate glycemic management. Similarities included number of patients that received initial insulin bolus $(p=1.000)$, accuracy of bolus dose $(p=0.666)$, accuracy of insulin infusion dose $(\mathrm{p}=1.000)$, accuracy of infusion to subcutaneous insulin transition $(\mathrm{p}=0.236)$, median glucose at time insulin infusion was stopped $(\mathrm{p}=0.850)$, accuracy of subcutaneous and intravenous insulin overlap $(p=0.427)$, and rates of hypoglycemia $(p=1.000)$. Of these outcomes, there was no statistically significant difference between the two groups (Table 2) (Table 3).

Patients without AGRO had a shorter time to gap of 15 (10hours and 56minutes vs. 11 hours and 27 minutes; $(\mathrm{p}=0.381)$ and a higher percentage of patients whose anion gap closed to 12 and remained $<15$ for rest of hospital stay ( $56 \%$ vs. $24 \%$; $=0.116)$. However, neither of these outcomes was statistically significant (Table 4).

Table I Baseline characteristics

\begin{tabular}{|c|c|c|c|}
\hline $\begin{array}{l}\text { Baseline characteristics } \\
(N=30)\end{array}$ & $\begin{array}{l}\text { Gap re-opening } \\
(n=21)\end{array}$ & $\begin{array}{l}\text { Without gap re-opening } \\
(n=9)\end{array}$ & $P$ value \\
\hline Median age (years) & $40(19-79)$ & $25(20-65)$ & 0.276 \\
\hline Male & $13(54 \%)$ & $4(44 \%)$ & 0.443 \\
\hline Median body mass index $\left(\mathrm{kg} / \mathrm{m}^{2}\right)$ & $28(19-65)$ & $27(18-47)$ & 0.715 \\
\hline Median weight $(\mathrm{kg})$ & $77(53-121)$ & $71(48-138)$ & 0.81 \\
\hline Median length of stay (days) & $4(I-14)$ & $3(I-9)$ & 0.313 \\
\hline First floor on admission & $\begin{array}{l}\text { Medicine or Step Down: } 20 \text { (95\%) } \\
\text { ICU: I (5\%) }\end{array}$ & $\begin{array}{l}\text { Medicine or Step Down: } 9 \text { (I00\%) } \\
\text { ICU: } 0(0 \%)\end{array}$ & I \\
\hline Type I diabetes mellitus & I5 (7|\%) & $5(56 \%)$ & 0.431 \\
\hline New onset diabetes & $4(19 \%)$ & $3(33 \%)$ & 0.64 \\
\hline Median $\mathrm{HgAlC}$ on admission & $12(8-18)$ & $12(12-18)$ & 0.63 \\
\hline Oral antidiabetic agent prescription at home & $2(10 \%)$ & I (II\%) & I \\
\hline Insulin prescription at home & $13(62 \%)$ & $3(33 \%)$ & 0.236 \\
\hline
\end{tabular}


Table Continued.

\begin{tabular}{lll}
$\begin{array}{l}\text { Baseline characteristics } \\
(\mathbf{N}=\mathbf{3 0})\end{array}$ & $\begin{array}{l}\text { Gap re-opening } \\
(\mathbf{n}=\mathbf{2} \mathbf{I})\end{array}$ & $\begin{array}{l}\text { Without gap re-opening } \\
(\mathbf{n}=\mathbf{9})\end{array}$ \\
\hline $\begin{array}{l}\text { Median serum blood glucose on admission }(\mathrm{mg} / \\
\mathrm{dL})\end{array}$ & $574(316-1250)$ & $492(301-621)$ \\
$\begin{array}{l}\text { Median anion gap upon admission } \\
\text { M }\end{array}$ & $27(18-49)$ & $22(20-26)$ \\
\hline
\end{tabular}

Table 2 Inpatient glycemic management

\begin{tabular}{|c|c|c|c|}
\hline Glycemic management $(\mathbf{N}=30)$ & $\begin{array}{l}\text { Gap re-opening ( } n \\
=2 \mathrm{I})\end{array}$ & $\begin{array}{l}\text { Without gap re-opening } \\
(n=9)\end{array}$ & $P$ value \\
\hline Received initial bolus & $8(38 \%)$ & $3(33 \%)$ & 1.000 \\
\hline $\begin{array}{l}\text { Of those that received an initial bolus, number that received } \\
\text { recommended dose ( } \pm 2 \text { units) }\end{array}$ & $5(62.5 \%)$ & $3(100 \%)$ & 0.666 \\
\hline Received recommended initial insulin infusion ( \pm 2 units) & $4(19 \%)$ & $2(22 \%)$ & 1.000 \\
\hline $\begin{array}{l}\text { Received long acting insulin within } 10 \% \text { of recommended OR home } \\
\text { regimen }\end{array}$ & $13(62 \%)$ & $3(33 \%)$ & 0.236 \\
\hline $\begin{array}{l}\text { Median last documented serum glucose prior to stopping insulin } \\
\text { infusion }\end{array}$ & $194(127-499)$ & $186(124-537)$ & 0.850 \\
\hline Intravenous and subcutaneous insulin overlap of at least I hour & $12(57 \%)$ & $3(33 \%)$ & 0.427 \\
\hline Documentation of discontinued IV insulin on the MAR & $15(7 \mid \%)$ & $6(66 \%)$ & 1.000 \\
\hline $\begin{array}{l}\text { Serum blood glucose }<70 \mathrm{mg} / \mathrm{dL} \text { at any point while on the insulin } \\
\text { infusion }\end{array}$ & I (5\%) & $0(0 \%)$ & 1.000 \\
\hline $\begin{array}{l}\text { Serum blood glucose }<70 \mathrm{mg} / \mathrm{dL} \text { at any point within } 24 \text { hours of } \\
\text { infusion completion }\end{array}$ & $0(0 \%)$ & $0(0 \%)$ & 1.000 \\
\hline
\end{tabular}

Table 3 Intravenous to subcutaneous insulin transition

\begin{tabular}{|c|c|c|c|}
\hline Subcutaneous insulin regimen for first 24 hours & Gap re-opening $(n=2 I)$ & Without gap re-opening $(n=9)$ & $P$ value \\
\hline & $21(100 \%)$ & $9(100 \%)$ & 1.000 \\
\hline \multirow{2}{*}{ Received Long Acting insulin Lantus NPH } & $19(90 \%)$ & $7(78 \%)$ & 0.563 \\
\hline & $2(10 \%)$ & $2(22 \%)$ & 0.563 \\
\hline Received meal-time (three times daily; regular or rapid) insulin & $13(62 \%)$ & $6(67 \%)$ & 1.000 \\
\hline Received sliding scale (regular or rapid) insulin & $21(100 \%)$ & $9(100 \%)$ & 1.000 \\
\hline
\end{tabular}

Table 4 Evaluation of anion gap closure

\begin{tabular}{|c|c|c|c|}
\hline Anion $\operatorname{gap}(\mathbf{N}=30)$ & $\begin{array}{l}\text { Gap re-opening } \\
(n=21)\end{array}$ & $\begin{array}{l}\text { Without gap re-opening } \\
(n=9)\end{array}$ & P value \\
\hline Median time to initial gap of 15 after start of insulin infusion & I Ihours 27 minutes & IOhours and 56 minutes & 0.381 \\
\hline $\begin{array}{l}\text { Initially gap closure to } \\
\text { Anion gap of } 12 \\
\text { Anion gap of } 15\end{array}$ & $\begin{array}{l}5(24 \%) \\
20(95 \%)\end{array}$ & $\begin{array}{l}5(56 \%) \\
9(100 \%)\end{array}$ & $\begin{array}{l}0.116 \\
1.000\end{array}$ \\
\hline $\begin{array}{l}\text { Median time to gap greater than } 15 \text { after initial closure to anion gap to } \\
\text { I5 }\end{array}$ & 19 hours and 12 minutes & N/A & N/A \\
\hline
\end{tabular}




\section{Discussion}

In 2016, a literature search of PubMed utilizing terms "diabetic ketoacidosis" and "anion gap" showed that current data available analyzes precipitating factors and baseline characteristics, ${ }^{6}$ novel management strategies, ${ }^{7-9}$ evaluation of factors leading to mortality, ${ }^{10,11}$ protocol and guideline validation and evaluation, ${ }^{12-18}$ and time to gap closure for diabetic ketoacidosis. ${ }^{19}$ However, none of the available data has analyzed factors leading to AGRO in patients with diabetic ketoacidosis. One study evaluated the impact of initiating subcutaneous insulin glargine within twelvehours of intravenous insulin infusion and found that this is a safe method for preventing future hyperglycemia without increased risk of hypoglycemia. ${ }^{20}$ In addition, a previous study evaluating pediatric patients in the intensive care unit saw DKA-induced anion gap resolution in 12.4-17.1 hours after the initiation of insulin therapy. ${ }^{21}$ In contrast, a 2014 study evaluating adult patients illustrated anion gap closure at approximately 10.3 hours. ${ }^{22}$ However, there are no studies that identify factors and patient characteristics leading to anion gap re-opening, and thus the purpose of this retrospective study. Our study illustrated that factors such as higher anion gap on admission, older age, and higher serum glucose may require more restrictive anion gap goals in order to improve patient outcomes and decrease length of stay.

There were multiple areas of guideline adherence and successful glycemic management. All patients received long-acting insulin and an insulin sliding scale and provider-adjusted insulin infusions resulted in low rates of hypoglycemia. In addition, the insulin infusion was stopped at the appropriate blood glucose. Our time to anion gap closure of 10.9-11.5hours was similar to the previously mentioned study that saw $10.3-10.4$ hours. ${ }^{22}$

There were multiple limitations to this study. First, the study was single-centered and utilized a small sample size of thirty patients. Second, the small sample size may have resulted in the study being under-powered and inhibited our ability to determine statistical significance of additional factors. Third, only serum blood glucose, not point of care blood glucose, was evaluated. This was done because serum blood glucose is more accurate than point of care blood glucose, however serum blood glucose is drawn less frequently than point of care blood glucose. This may have altered our results of initial glucose on admission and glucose at the time the insulin infusion was stopped. Another limitation included if stop time of the insulin infusion was not documented, then the last rate change was recorded as the insulin infusion stop time. This inhibited our ability to assess accurate transition from infusion to subcutaneous insulin therapy. Also, additional insulin intravenous pushes outside of the bolus and basal infusion were not evaluated and thus could have contributed to a more rapid anion gap closure. In addition, we were unable to assess outpatient adherence with diabetes regimen. And finally, the recommended subcutaneous insulin doses were assessed based on our institution's guideline for transition from intravenous to subcutaneous insulin, which may be different than other institutions.

\section{Conclusion}

In conclusion, a higher anion gap on admission is a significant predictor of AGRO. This study has identified multiple other factors that may have clinical significance in predicting AGRO such as increased age and increased admission blood glucose. Patients with these factors may require stricter anion gap goals during treatment of DKA. Providers should utilize ADA guidelines as well as incor- porate patient factors into their decision making when determining what patients may require a stricter anion gap goal. Since adhering to the ADA guidelines improves patient outcomes, institutions should evaluate their current $\mathrm{CPOE}$ system and evaluate their normalized lab values. Additional large multi-center studies are needed to further validate these potential factors.

\section{Acknowledgements}

None.

\section{Conflict of interest}

Author declares that there is no conflict of interest.

\section{References}

1. Kitabchi AE, Umpierrez GE, Miles JM, et al. Hyperglycemic crises in adult patients with diabetes. Diabetes Care. 2009;32(7):1335-1343

2. Gosmanov AR, Gosmanova EO, Dillard-cannon E. Management of adult diabetic ketoacidosis. Diabetes Metab Syndr Obes. 2014;7:255-264.

3. Kitabchi AE, Umpierrez GE, Miles JM, et al. Hyperglycemic crises in adult patients with diabetes. Diabetes Care. 2009;32(7):1335-1343.

4. Westerberg DP. Diabetic ketoacidosis: evaluation and treatment. Am Fam Physician. 2013;87(5):337-346.

5. Kitabchi AE, Umpierrez GE, Miles JM, et al. Hyperglycemic crises in adult patients with diabetes. Diabetes Care. 2009;32(7):1335-1343.

6. Qari F. Clinical characteristics of patients with diabetic ketoacidosis at the Intensive Care Unit of a University Hospital. Pak J Med Sci. 2015;31(6):1463-1466.

7. Ullal J, Mcfarland R, Bachand M, et al. Use of a Computer-Based Insulin Infusion Algorithm to Treat Diabetic Ketoacidosis in the Emergency Department. Diabetes Technol Ther. 2016;18(2):100-103.

8. Doshi P, Potter AJ, De los santos D, et al. Prospective randomized trial of insulin glargine in acute management of diabetic ketoacidosis in the emergency department: a pilot study. Acad Emerg Med. 2015;22(6):657-662.

9. Goyal N, Miller JB, Sankey SS, et al. Utility of initial bolus insulin in the treatment of diabetic ketoacidosis. J Emerg Med. 2010;38(4):422-427.

10. Suwarto S, Sutrisna B, Waspadji S, et al. Predictors of five days mortality in diabetic ketoacidosis patients: a prospective cohort study. Acta Med Indones. 2014;46(1):18-23.

11. Guisado-vasco P, Cano-megías M, Carrasco-de la fuente M, et al. Clinical features, mortality, hospital admission, and length of stay of a cohort of adult patients with diabetic ketoacidosis attending the emergency room of a tertiary hospital in Spain. Endocrinol Nutr. 2015;62(6):277-284.

12. Evans KJ, Thompson J, Spratt SE, et al. The implementation and evaluation of an evidence-based protocol to treat diabetic ketoacidosis: a quality improvement study. Adv Emerg Nurs J. 2014;36(2):189-98.

13. Westerberg DP. Diabetic ketoacidosis: evaluation and treatment. $\mathrm{Am}$ Fam Physician. 2013;87(5):337-346.

14. Arora S, Probst MA, Agy C, et al. Point-of-care beta-hydroxybutyrate testing for assessing diabetic ketoacidosis severity prior to treatment in the emergency department. Diabetes Res Clin Pract. 2011;94(3):e86-88.

15. Maciel AT, Park M. A physicochemical acid-base approach for managing diabetic ketoacidosis. Clinics (Sao Paulo). 2009;64(7):714-718.

16. Ferreri R. Treatment practices of diabetic ketoacidosis at a large teaching hospital. J Nurs Care Qual. 2008;23(2):147-154 
17. Bull SV, Douglas IS, Foster M, et al. Mandatory protocol for treating adult patients with diabetic ketoacidosis decreases intensive care unit and hospital lengths of stay: results of a nonrandomized trial. Crit Care Med. 2007;35(1):41-46.

18. Eledrisi MS, Alshanti MS, Shah MF, et al. Overview of the diagnosis and management of diabetic ketoacidosis. Am J Med Sci. 2006;331(5):243-251.

19. Nemecek BD, Hermayer KL, Arnold PC, et al. Evaluation of Ward Management of Diabetic Ketoacidosis. Clin Diabetes. 2014;32(3):100-104.
20. Hsia E, Seggelke S, Gibbs J, et al. Subcutaneous administration of glargine to diabetic patients receiving insulin infusion prevents rebound hyperglycemia. J Clin Endocrinol Metab. 2012;97(9):3132-3137.

21. Shankar V, Haque A, Churchwell KB, et al. Insulin glargine supplementation during early management phase of diabetic ketoacidosis in children. Intensive Care Med. 2007;33(7):1173-1178.

22. Nemecek BD, Hermayer KL, Arnold PC, et al. Evaluation of Ward Management of Diabetic Ketoacidosis. Clin Diabetes. 2014;32(3):100-104. 